Perspective Article

\title{
Hybrid cellulose-poly(4-vinylpyridine) adsorbents produced via ATRP and their application to target polyphenols in winemaking, olive oil production and almond processing residues
}

\author{
C.P. Gomes ${ }^{a}$, R.C.S. Dias ${ }^{a, *}$, M.R.P.F.N. Costa ${ }^{b}$ \\ ${ }^{a}$ Centro de Investigação de Montanha (CIMO), Instituto Politécnico de Bragança, Campus de Santa Apolónia, 5300-253 Bragança, Portugal \\ ${ }^{\mathrm{b}}$ LSRE, Faculdade de Engenharia da Universidade do Porto, Rua Roberto Frias s/n, 4200-465 Porto, Portugal
}

\section{A R T I C L E I N F O}

\section{Keywords:}

Hybrid materials

Cellulose-synthetic

ATRP grafting

Adsorbents

Downstream processing

\begin{abstract}
A B S T R A C T
Hybrid cellulose-poly(4-vinylpyridine) particles (MCC-P(4VP)) were synthesized via ATRP grafting and used as adsorbents to target polyphenols in residues derived from winemaking, olive oil production and almond processing. These hybrid particles preserve good conditions to be used in continuous sorption/desorption processes and were assessed for the retention of polyphenols in solvent mixtures ranging from 0 to $100 \%$ water content. Other seven adsorbents were used in these assays, including three commercial resins and further materials synthesized in this research with 4VP.

We show that the 4VP based adsorbents present a much higher average polyphenol retention in a broad range of working conditions, namely concerning the water content of the extract. This behavior stems from the strong binding capacity of the pyridyl functional group towards many polyphenols and excels with MCC-P(4VP) adsorbents when the hydrophobic interactions play a minor role.

MCC-P(4VP) adsorbents were also used for purification and fractionation of polyphenols using simple sorption/desorption steps. A fraction enriched with the malvidin-3-O-glucoside anthocyanin was obtained from grape pomace. Fractions with high content of isorhamnetin-3-O-rutinoside were isolated from almond. Fractions enriched in secoiridoids and flavonoids were produced from olive mill wastewater while many phenolic alcohols and acids were removed. These results show the potential of the MCC-P(4VP) adsorbents to improve downstream processing of polyphenols and increase the biorefining efficiency, namely through the direct handling of organic extracts at high polyphenol concentration.

This research also has disclosed the formation of a polymer population deviating from the expected homogeneous process in ATRP grafting. The partition of ATRP reactants between two regions in the homogeneous phase could be a source of some potentially interesting issues relevant to monomers presenting strong H-bond capability, such as 4VP. Thus, many future developments are possible for the designing of the functional materials here addressed, including the introduction of molecular recognition features.
\end{abstract}

\section{Introduction}

Development of tailored adsorbents is an important issue for different application fields, ranging from analytical procedures to large scale industrial processes. High specificity/selectivity towards target compounds, high retention capacities and ease of regeneration are key parameters to evaluate adsorbents performance. At the end, the triangulation between the kind of adsorbent, target solute(s) and solvent matrices will determine the efficiency of the aimed sorption/desorption process. For relatively simple systems the interactions between adsorbent/adsorbate(s)/solvent(s) can be described/predicted by molecular simulation but real samples are difficult to handle with these tools due to the heterogeneity of common adsorbents and the complexity of the mixtures solute/solvent intervenient in the sorption/desorption processes. Therefore, some kind of semi-empirical approach is often used to design new adsorbents.

Among different kinds of adsorbents (e.g. activated carbons, minerals, lignocellulosic/polysaccharides), synthetic resins present important advantages, namely their durability, stability, ease of regeneration and the possibility to create tailored active materials. Indeed, some

\footnotetext{
* Corresponding author.

E-mail address: rdias@ipb.pt (R.C.S. Dias).
} 
classes of polymeric synthetic resins (e.g. Amberlite ${ }^{\circledR}$, Supellite ${ }^{\mathrm{TM}}$ or Reillex $^{\mathrm{TM}}$ resins) are produced at large scale to be used in a broad range of application fields (environmental protection, biotechnology, pharmaceutics, etc). Improvement in specificity and capacity for the adsorption of selected compounds can be achieved with synthetic polymeric resins using synthesis routes allowing the functionalization of the materials or even the introduction of molecular recognition capabilities through molecular imprinting techniques. Classical free radical polymerization (FRP) and controlled radical polymerization (CRP) mechanisms play a key role for the tailoring of new adsorbent polymer networks. Moreover, the production of hybrid materials, incorporating natural and synthetic polymers, is also possible with appropriated synthesis routes, namely CRP, allowing the addressing of eco-sustainability requirements with the inclusion of the natural materials (e.g. cellulose).

Due to the relatively simple operation requirements, low cost and prevention of the degradation of the target compounds, sorption/ desorption is a commonly used method to target polyphenols in plant extracts. In practice, two general classes of processes are found in this application field:

i) The recovery of target polyphenols from plant extracts to be used as high-added value products in food, cosmetic, pharmaceutical or chemical industries.

ii) The treatment of effluents generated in industries involving the processing of plant extracts (generally wastewater effluents). The treatment of olive mill wastewater is an important issue in this context.

A major difficulty to be addressed with the sorption/desorption of plant extracts is the high complexity of the mixtures inherent to these systems that include a plethora of different compounds. Moreover, extracts composition is strongly dependent on their production conditions, namely the kind(s) of solvent used. Therefore, the development of tailored adsorbents is a key point aiming at the design of efficient sorption/desorption processes to target polyphenols.

This research explores the tailoring of functional adsorbents considering the grafting of synthetic polymers in cellulose backbone through Atom Transfer Radical Polymerization (ATRP). Actually, the grafting of cellulose with synthetic polymers through the use of ATRP, other CRP techniques, or free radical polymerization is being considered in the scientific community as an effective route to generate new materials for many different applications. Ions removal from aqueous solutions, dyes degradation, stimuli-responsive hydrogels fabrication or aqueous aggregates generation [1-5], are just a few examples of such kinds of applications (ref. [1] contains a comprehensive list of cellulose modification techniques and applications). A significant change in the native cellulose properties can be achieved with the grafting of synthetic polymers in this abundant and biodegradable material, thus increasing sustainability.

Additionally, nitrogen containing polymers, especially those based on vinylpyridine monomers [6], present enhanced adsorption capabilities towards specific compounds, which justifies their use for the tailoring of some kinds of advanced materials. Indeed, due to their chelating capability, 4-vinylpyridine based materials are being considered for heavy metals adsorption with applications in wastewater/industrial effluents treatment [7-10], enzymes/protein adsorption $[11,12]$ and other applications involving biomedicine or catalysis [13]. Due to the potential strong interaction between 4-vinylpyridine polymers and many polyphenols, our research explores hybrid cellulose-poly (4-vinylpyridine) materials to target these classes of compounds. As far as is of our knowledge, this approach is considered for the first time with the processing of real plant extracts.

The materials here synthesized are assessed to target polyphenols in winemaking, olive oil and almond industries that are three important agricultural/industrial sectors with worldwide expression and particular joint relevance in the Mediterranean area. We show that hybrid cellulose-poly(4-vinylpyridine) adsorbents present a very good performance for polyphenols adsorption in a broad range of working conditions with extracts of these three different plants, namely considering diverse solvents (e.g. with high organic content mixtures and with wastewater effluents). Indeed, the materials synthesized include a microcrystalline cellulose core $(20 \mu \mathrm{m})$ preserving good conditions to be used in continuous sorption/desorption processes and excels for polyphenols retention when hydrophobic effects play a minor role due to functionalization with the pyridyl group. Thus, more flexible and efficient downstream processing of polyphenols can be designed with these adsorbents.

\section{Materials and methods}

\subsection{Reagents}

Microcrystalline cellulose (MCC, powder, $20 \mu \mathrm{m}$, cotton linters), $\alpha$-bromoisobutyryl bromide (BIBB, 98\% purity), triethylamine (TEA, 99.5\% purity), 4-(dimethylamino)pyridine (DMAP, purity $\geq 99 \%$ ), ethyl 2-bromopropionate (EBrP, 99\% purity), $\mathrm{CuBr}$ (97\% purity) and $\mathrm{N}, \mathrm{N}, \mathrm{N}^{\prime}$, $\mathrm{N}^{\prime \prime}, \mathrm{N}^{\prime \prime}$-pentamethyldiethylenetriamine (PMDETA, 99\% purity) were purchased from from Sigma-Aldrich. Acrylonitrile (AN, 99\% purity) and 4-vinylpyridine (4VP, 95\% purity) were provided by Alfa Aesar. Analytical reagent grades for acetonitrile (ACN), dimethylformamide (DMF), acetic acid $(\mathrm{AcOH})$, and methanol $(\mathrm{MeOH})$ were bought from Fisher Scientific and for ethanol (EtOH) from PanReac. Millipore water (Milli-Q quality) was used in all the experiments unless otherwise mentioned.

\subsection{Immobilization of $\alpha$-bromoisobutyryl bromide (BIBB) in microcrystalline cellulose (MCC)}

The immobilization of BIBB in MCC was performed using the reported esterification procedure [14,15]. Typically, $1 \mathrm{~g}$ of MCC was dispersed in DMF $(100 \mathrm{~mL})$ using sonication. Then, triethylamine $(8 \mathrm{~mL})$ and DMAP ( $4 \mathrm{~g}$ ) were added to the MCC dispersion and, afterwards, BIBB $(8 \mathrm{~mL})$ was dropwise fed to the suspension at $0{ }^{\circ} \mathrm{C}$. The reaction was allowed to proceed at room temperature during $24 \mathrm{~h}$. At the end, ethanol was used to terminate the esterification. Products were washed with DMF until achievement of a colorless liquid phase and then ATRP macroinitiator MCC-Br was purified using dialysis with deionized water, followed by drying in vacuum oven at $40^{\circ} \mathrm{C}$.

\subsection{Grafting of synthetic polymer chains in MCC through ATRP}

$\mathrm{CuBr}(5 \mathrm{mg})$ was dissolved in DMF ( $2 \mathrm{~mL}$ ) via sonication. Then, MCC$\operatorname{Br}(50 \mathrm{mg})$, PMDETA $(20 \mu \mathrm{L}), \operatorname{EBrP}(40 \mu \mathrm{L}), 4 \mathrm{VP}(3.25 \mathrm{~mL})$ were added to the $\mathrm{CuBr}$ solution. The resulting solution was purged with a flow of dry argon for $30 \mathrm{~min}$ and the polymerization took place during $24 \mathrm{~h}$ at $60{ }^{\circ} \mathrm{C}$. Acrylonitrile polymer chains were grafted in MCC following a similar procedure using AN instead of 4VP. The final products (MCC-Br4VP or MCC-Br-AN) were washed with DMF and $\mathrm{MeOH}$ and dried in vacuum oven. Through these purification procedures, hybrid MCCsynthetic particles (DMF insoluble) and free polymer (methanol insoluble) were isolated and the monomer conversion as well as the grafting ratio were gravimetrically estimated.

\subsection{Products characterization using FT-IR spectroscopy}

Purified and dried products were characterized through FT-IR spectroscopy with a Perkin Elmer, model Spectrum Two ${ }^{\mathrm{TM}}$, instrument. These analysis were directly performed in ATR mode or, when needed, polymers were mixed with $\mathrm{KBr}$ and pressed into pellets in order to collect the correspondent IR spectra. 


\subsection{Polymer characterization using SEC/RI/LALS/RALS}

SEC analysis was considered to get information concerning the structure of different soluble polymers synthesized in this research. The used SEC apparatus is composed of a Viscotek GPCmax VE 2001 pumping system with Refractive Index (RI), Low Angle Light Scattering (LALS) and Right Angle Light Scattering (RALS) detection. Polymers were fractionated in a train of two columns PLgel $5 \mu \mathrm{m}$ Mixed-D+ PLgel $3 \mu \mathrm{m}$ Mixed-E with dimensions $\mathrm{L} \times \mathrm{D}=300 \mathrm{~mm} \times 7.5 \mathrm{~mm}$. DMF with $20 \mathrm{mM} \mathrm{LiBr}$ was used as eluent and the measurements were performed at $50{ }^{\circ} \mathrm{C}$ and $\mathrm{Q}=0.5 \mathrm{~mL} / \mathrm{min}$.

\subsection{Preparation of different kinds of almond residues and grape pomace extracts}

Almond fruits, including the hull, shell and the nuts were collected in Torre de Moncorvo (Trás-os-Montes, Portugal) during the autumn of 2019. After fruit components separation and drying, hull and almond shell were milled and used in Soxhlet extraction considering different runs with EtOH/water 80/20, ACN/DMF 50/50 and EtAc/EtOH 50/50 $(v / v)$ solvents. Soxhlet extractions were performed using a ratio of $30 \mathrm{~g}$ of dried mass per $200 \mathrm{~mL}$ of solvent.

Grape pomace was obtained in the autumn of 2019, directly from a red wine producer located at Vila Real (Douro region, Portugal). Grape pomace before and after distillation (for brandy production) were both considered as polyphenols sources. After drying, grape pomace was also milled and used in Soxhlet extraction following the same procedures above described for almond residues.

All the extracts produced were filtered through a $0.45 \mu \mathrm{m}$ nylon filter before use in sorption/desorption assays.

\subsection{Aqueous effluents derived from the almond blanching process and olive mill wastewater}

The almond blanching process was performed boiling $230 \mathrm{~g}$ of kernel fruits with skin in $700 \mathrm{~mL}$ of deionized water during $1 \mathrm{~min}$. The liquid extract was filtered to remove solid mass and then used in sorption/ desorption assays.

Olive mill wastewater was collected in winter of 2019 from an olive oil producer in Moura (Alentejo, Portugal) and filtered to remove suspended solids before use in sorption/desorption processes.

\subsection{Sorption/desorption experiments}

For assessment of the sorption/desorption capabilities of the different adsorbents considered in this work, $200 \mathrm{mg}$ of the dry materials were used in solid phase extraction (SPE) packing cartridges. Adsorbents were first cleaned (successive loading and elution steps using methanol/ acetic acid 90/10 with UV monitoring) and afterwards conditioned with the desired testing solvent during at least $24 \mathrm{~h}$ before use. Cartridges were then loaded with $5 \mathrm{~mL}$ of the selected extract considering typically $15 \mathrm{~min}$ for total percolation time. The adsorbed polyphenol profile was quantified through HPLC-DAD analysis of the liquid collected at column outlet (UV/vis monitoring was also considered for global absorption quantification). Adsorbent saturation runs were also performed by continuous feeding of the column with the selected extract, up to achievement of a constant UV response at column outlet.

Recovery of the adsorbed compounds was assessed by collecting fractions with different elution solvents, ranging from washing with the same solvent used for loading, up to the use of stronger displacement mixtures (e.g. methanol/acetic acid 90/10). The effect of the temperature in polyphenols desorption was also assessed by changing the temperature of the elution solvents (e.g. room temperature up to $50{ }^{\circ} \mathrm{C}$ ). Phenolic profile of the recovered fractions was measured using HPLCDAD analysis.

\subsection{Polyphenols identification/quantification through gradient HPLC- $D A D$}

A Jasco MD-4010 photo diode array (PDA) detector was used to carry out the HPLC-DAD analyses. Nucleosil ${ }^{\circledR}$ C18 analytical column, $\mathrm{L} \times \mathrm{I}$.D. $=15 \mathrm{~cm} \times 4.6 \mathrm{~mm}$ and $5 \mu \mathrm{m}$ particle size, working at room temperature, has been chosen. The flow rate was $1 \mathrm{~mL} / \mathrm{min}$ and the mobile phases used for chromatographic analysis were: (A) acetonitrile/water (10/90) at $\mathrm{pH}=3$ (adjusted with acetic acid) and (B) acetonitrile/water (90/10), also at $\mathrm{pH}=3$. The linear gradient method was used, starting with $100 \%$ of solvent A and ending with $100 \%$ of solvent B. Previous results of our research with plant extracts analysis through LC-DAD-ESI-MS ${ }^{\mathrm{n}}$ [16-18] were also considered in the present work for polyphenols identification.

\subsection{Measurement of the bromide content in the MCC immobilized with $B I B B$}

These bromine determinations were performed by ICP-MS (Inductively Coupled Plasma - Mass Spectrometry) using a Thermo Scientific ${ }^{\mathrm{TM}}$ iCAP $^{\mathrm{TM}} \mathrm{Q}$ instrument at LAQV/REQUIMTE - Laboratory of Applied Chemistry (Trace Element Analysis unit), Faculty of Pharmacy of the University of Porto (FFUP). To perform these analyses, MCC-Br was first dissolved in dimethyl sulfoxide at $70{ }^{\circ} \mathrm{C}$ at a concentration of $0.675 \mathrm{mg}$ / $\mathrm{mL}$.

\subsection{SEM analysis of the adsorbent particles}

Scanning Electron Microscopy (SEM) characterization of the particles involved in this research was performed at the International Iberian Nanotechnology Laboratory (INL), Braga, using the FIB/SEM system HELIOS Nanolab 450S. SEM imaging was obtained using an electron beam of $3 \mathrm{keV}$, beam current $25 \mathrm{pA}$ and field free lens mode.

\subsection{Measurement of the surface area and pore volume of the adsorbents}

The Brunauer-Emmett-Teller (BET) specific surface area and pore volume of the produced adsorbent particles were determined through $\mathrm{N}_{2}$ adsorption/desorption isotherms at $77 \mathrm{~K}$ using a Quantachrome NOVA 4200e adsorption analyzer.

\section{Results and discussion}

Fig. 1 depicts the synthetic steps considered to get hybrid cellulosesynthetic materials via ATRP. Our approach follows the same methodology described in previous related works [5,14,15]. At a first stage (Fig. 1(a)), BIBB was immobilized in microcrystalline cellulose (MCC) through the reaction with $\mathrm{OH}$ groups in a DMF mixture containing TEA and DMAP (see Section 2.2 for the detailed experimental conditions used). Confirmation of the BIBB immobilization in MCC, generating the ATRP macro-initiator MCC-Br, was performed using FTIR, as shown in Fig. 2. Comparison of the FTIR spectra for native MCC and purified MCC$\mathrm{Br}$ shows clearly the emergence of a peak in MCC-Br $\left(1740 \mathrm{~cm}^{-1}\right)$ correspondent to the stretching of the carbonyl group $(-\mathrm{C}=\mathrm{O})$ resulting from the reaction between $\mathrm{OH}$ groups and BIBB (see also Fig. 1(a)). The $\mathrm{Br}$ content in the macro-initiator MCC-Br was measured by ICP-MS and the concentration of $135 \mathrm{mg} / \mathrm{g}$ was obtained, which is in the same range of measurements reported in previous related works (see [15] and references therein).

The synthesized ATRP macro-initiator was afterwards used to graft synthetic polymer chains in MCC, namely $\mathrm{P}(4 \mathrm{VP})$ and PAN chains, as depicted in Fig. 1(b), considering the ATRP of 4VP for illustration purposes. Besides the ATRP macro-initiator, this synthesis step included also the use of EBrP initiator aiming at inducing the formation of free synthetic polymer in addition to the grafted chains. This procedure is often exploited to get indirect information concerning the structure of the grafted-polymer through the analysis of the free product $[14,19]$. 
(a)
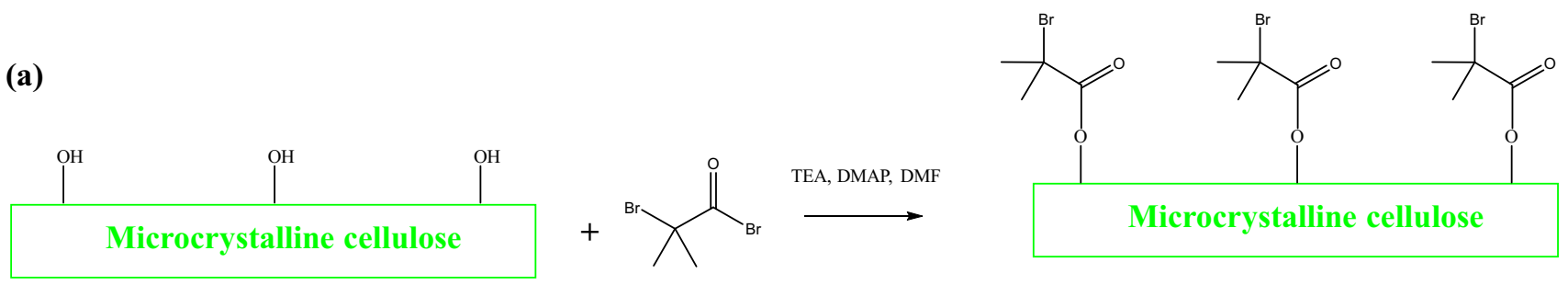

(b)
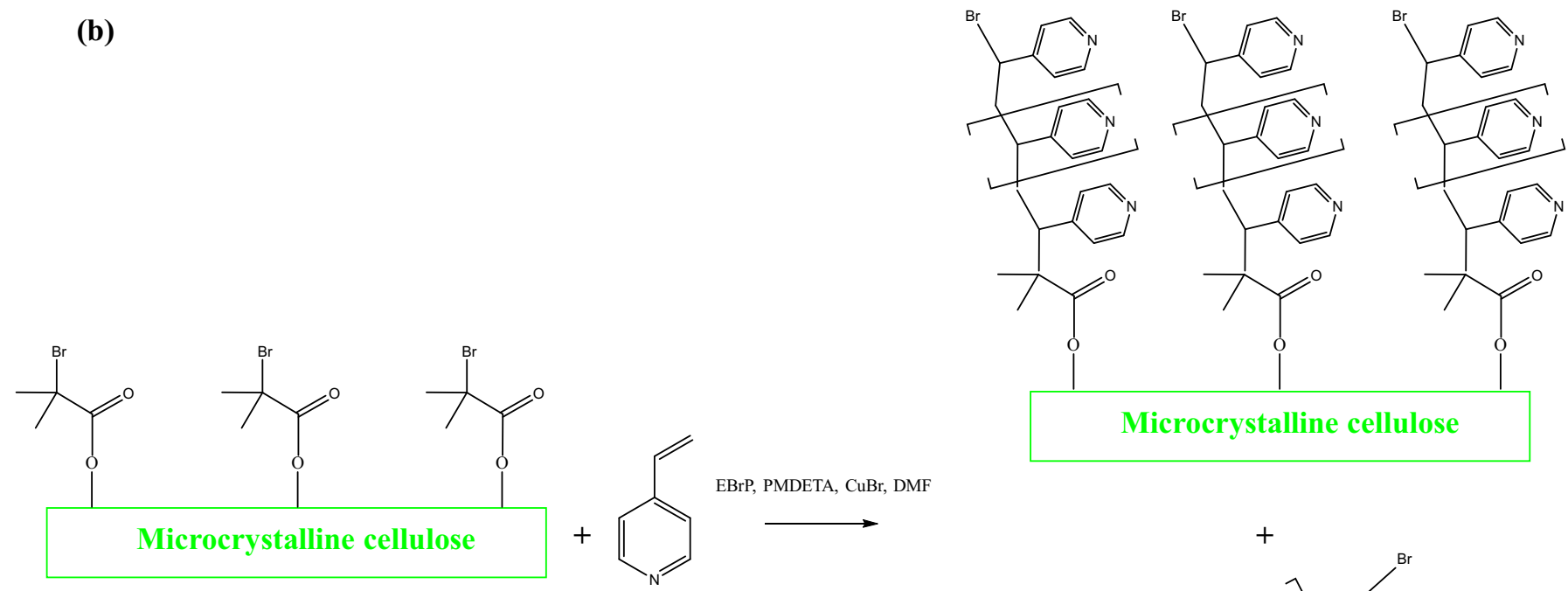

Microcrystalline cellulose

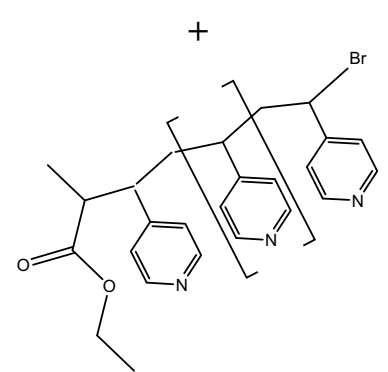

Fig. 1. Depiction of (a) immobilization of $\alpha$-bromoisobutyryl bromide (BIBB) in microcrystalline cellulose (MCC) with production of the ATRP macro-initiator MCC$\mathrm{Br}$ and (b) grafting of 4VP polymer chains in MCC through ATRP. In the grafting step, besides the macro-initiator MCC-Br, the ATRP initiator EBrP was also included to generate the formation of free $\mathrm{P}(4 \mathrm{VP})$ homopolymer.

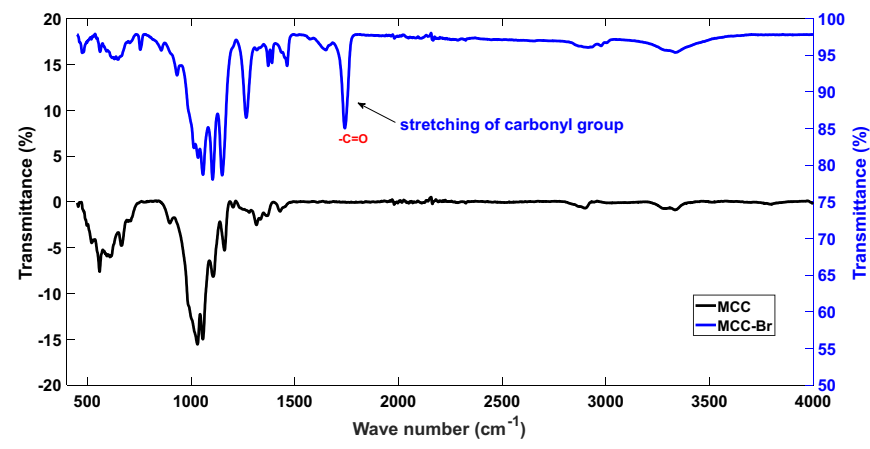

Fig. 2. FTIR spectra for the native microcrystalline cellulose (MCC) and for the purified product resulting from the immobilization of $\alpha$-bromoisobutyryl bromide in MCC (MCC-Br).

With the systems here considered, both free $\mathrm{P}(4 \mathrm{VP})$ and PAN formed in these grafting ATRP runs are soluble in the DMF liquid phase while the cellulose containing products (starting with MCC-Br up to the MCC-P (4VP) or MCC-PAN hybrid materials) remain insoluble along the reaction (the heterogeneous approach was considered). Table 1 contains detailed information concerning the synthesis conditions used to generate the MCC-P(4VP) and MCC-PAN hybrid adsorbents.
Table 1

Reaction conditions used to synthesize different ATRP and FRP products considered for the development of hybrid cellulose-poly(4-vinylpyridine) and cellulose-polyacrylonitrile adsorbents. All the polymerizations were performed with DMF as solvent.

\begin{tabular}{|c|c|c|c|c|c|c|}
\hline Product & $\begin{array}{l}\text { MCC-Br } \\
\text { (g/L) }\end{array}$ & $\begin{array}{l}\text { EBrP } \\
\text { (mol/ } \\
\text { L) }\end{array}$ & $\begin{array}{l}\text { Monomer } \\
\text { (mol/L) }\end{array}$ & $\begin{array}{l}\mathrm{CuBr} \\
\text { (mol/ } \\
\text { L) }\end{array}$ & $\begin{array}{l}\text { PMDETA } \\
(\mathrm{mol} / \mathrm{L})\end{array}$ & $\begin{array}{l}\text { AIBN } \\
\text { (mol/ } \\
\text { L) }\end{array}$ \\
\hline $\begin{array}{l}\text { MCC-P } \\
\text { (4VP) }\end{array}$ & 9.511 & $\begin{array}{l}5.749 \\
\times 10^{-2}\end{array}$ & 5.752 & $\begin{array}{l}6.630 \\
\times 10^{-3}\end{array}$ & $\begin{array}{l}1.804 \times \\
10^{-2}\end{array}$ & - \\
\hline $\begin{array}{l}\text { MCC- } \\
\text { PAN }\end{array}$ & 12.365 & $\begin{array}{l}7.519 \\
\times 10^{-2}\end{array}$ & 7.521 & $\begin{array}{l}8.569 \\
\times 10^{-3}\end{array}$ & $\begin{array}{l}2.359 \times \\
10^{-2}\end{array}$ & - \\
\hline $\mathrm{P}(4 \mathrm{VP})$ & - & $\begin{array}{l}5.738 \\
\times 10^{-2}\end{array}$ & 5.709 & $\begin{array}{l}7.114 \\
\times 10^{-3}\end{array}$ & $\begin{array}{l}1.787 \times \\
10^{-2}\end{array}$ & - \\
\hline PAN & - & $\begin{array}{l}7.522 \\
\times 10^{-2}\end{array}$ & 7.522 & $\begin{array}{l}8.347 \\
\times 10^{-3}\end{array}$ & $\begin{array}{l}2.257 \times \\
10^{-2}\end{array}$ & - \\
\hline $\begin{array}{l}\text { P(4VP) - } \\
\text { FRP }\end{array}$ & - & - & 3.478 & - & - & $\begin{array}{l}1.427 \\
\times 10^{-2}\end{array}$ \\
\hline $\begin{array}{l}\text { PAN - } \\
\text { FRP }\end{array}$ & - & - & 3.440 & - & - & $\begin{array}{l}6.900 \\
\times 10^{-3}\end{array}$ \\
\hline
\end{tabular}

Gravimetrically measured content of P(4VP) in the final MCC-P(4VP) product was $90 \%$, while $64 \%$ of PAN was estimated for the MCC-PAN hybrid material. A high incorporation rate of the synthetic polymer in the hybrid materials was here explored in order to compare the 
adsorption capabilities of the modified materials relatively to cellulose. However, much lower synthetic contents (e.g. lower than 10\%) can be attained depending on the materials applications by changing the grafting conditions. Incorporation of the synthetic polymers in the final materials was also confirmed using FTIR analysis, as shown in Figs. 3 and 4. Assignments corresponding to the $-\mathrm{C}=\mathrm{N}\left(1415 \mathrm{~cm}^{-1}\right)$ and $\mathrm{C}=\mathrm{C}$ (1550 and $1600 \mathrm{~cm}^{-1}$ ) pyridyl groups are clearly observable in the spectrum collected for the MCC-P(4VP) materials, while the nitrile group $(-\mathrm{C} \equiv \mathrm{N}$ ) assignment is patent with the FTIR analysis for MCC-PAN $\left(2245 \mathrm{~cm}^{-1}\right)$.

In Figs. 5 and 6 are presented the SEC/RI/LALS/RALS traces observed for the free $\mathrm{P}(4 \mathrm{VP})$ and PAN polymers collected at the end of the grafting reactions leading to the formation of the MCC-P(4VP) and MCC-PAN hybrid materials, respectively. For comparison purposes, these plots include also the SEC traces for P(4VP) and PAN homopolymers synthesized via ATRP with EBrP initiation, as well as for samples of the same homopolymers produced by free radical polymerization (FRP) with AIBN initiation. The reaction conditions used in the synthesis of these four homopolymers can be found in Table 1 . For the interpretation of the results in Figs. 5 and 6, it should be noted that the chromatogram traces appearing after the system peak (starting at around 18 min elution time) are due to non-polymer species, namely 4VP monomer and its possible metal coordination complexes (this effect is not pronounced with the AN monomer).

Results presented in Fig. 5(a) show the formation of a bimodal polymer population for the free polymer produced during the MCC-P (4VP) grafting process. Such bimodal polymer population is not observed for the $\mathrm{P}(\mathrm{VPP})$ homopolymer synthesized in a common ATRP process with $\mathrm{EBrP} / \mathrm{CuBr} / \mathrm{PMDETA}$ (see Table 1), as presented in Fig. 5 (b). An unimodal polymer population is also formed when the $\mathrm{P}(4 \mathrm{VP})$ homopolymer is synthetized via FRP, as shown in Fig. 5(c). The comparison between the bimodal polymer correspondent to the free chains formed in MCC-P(4VP) grafting and the unimodal polymers produced via ATRP or FRP homopolymerizations is highlighted in Fig. 5(d). This comparison shows that the free polymer formed during the grafting process is composed of a population close to that formed in a similar homogeneous ATRP process and another with very high molecular size, even higher than that found in FRP synthesis. Thus, a second free polymer population is formed during the grafting process in conditions different from those expected with the homogenous conditions.

The formation of a bimodal polymer population is also observed for the free polymer with the ATRP grafting process involving the AN monomer, as presented in Fig. 6(a). Again, as expected, unimodal populations were measured for PAN homopolymers synthesized in a common ATRP process with EBrP/CuBr/PMDETA or via FRP, as shown in Fig. 6(b-c). The comparison in Fig. 6(d) highlights again that the free polymer in grafting is composed of a population similar to that formed with an analogous homogeneous ATRP, plus another population with

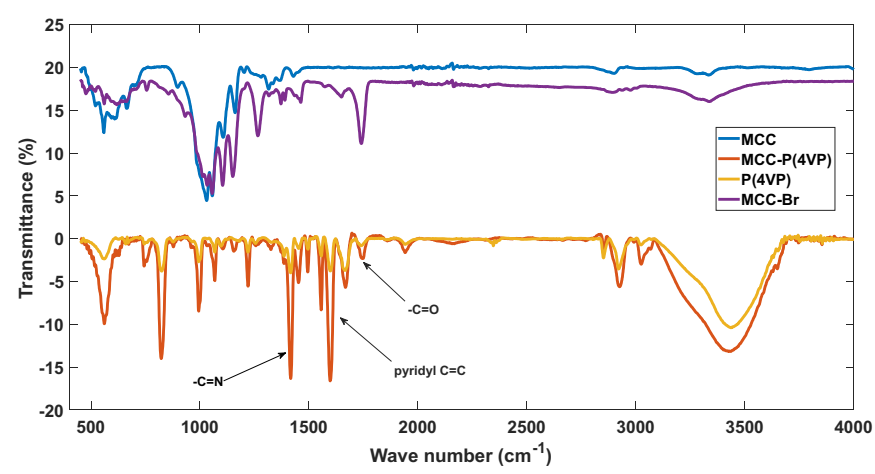

Fig. 3. FTIR spectra for MCC, MCC-Br, ATRP synthesized hybrid cellulose-poly (4-vinylpyridine) (MCC-P(4VP)) and an ATRP synthesized P(4VP) homopolymer.

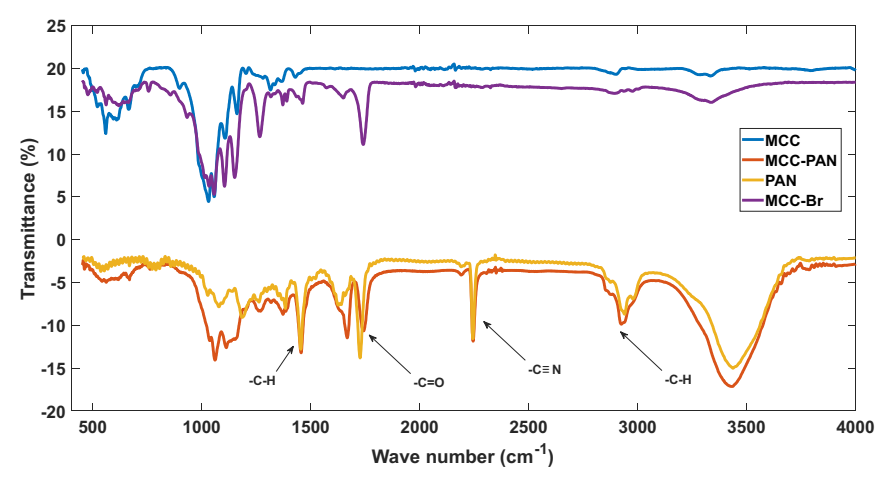

Fig. 4. FTIR spectra for MCC, MCC-Br, ATRP synthesized hybrid cellulosepolyacrylonitrile (MCC-PAN) and an ATRP synthesized PAN homopolymer.

much higher molecular size. This second population is less pronounced in the grafting with AN than that observed with $4 \mathrm{VP}$, as becomes evident through the comparison of Figs. 5(d) and 6(d).

The formation of multimodal polymer populations can be found in non-linear polymerization systems due to variable degree of branching/ crosslinking among the different species [20,21]. Following a different mechanism, the bimodal populations above described for the free polymer formed during MCC-P(4VP) and MCC-PAN synthesis should be a consequence of the coexistence of two regions with different reaction conditions in the liquid phase. In the bulk of the DMF liquid phase the reactant concentrations should be closer to that correspondent to a homogeneous ATRP, while in the vicinity of the solid phase (cellulose + grafted polymer) these concentrations can be substantially different. Dissimilar adsorption of the different ATRP reactants in the solid phase should lead to the formation of a polymer population not following the expected homogeneous process. This issue is magnified when $\mathrm{P}(4 \mathrm{VP})$ is involved due to the inherent strong H-bond capability and ability to generate metal coordination complexes. The issues just reported with the free polymer structure anticipate that the distribution of synthetic brushes grafted in cellulose should also deviate from the one expected in homogeneous ATRP products.

In order to further investigate the formation of these bimodal polymer populations, other ATRP grafting process were performed with 4VP and AN, changing the concentration of MCC-Br macro-initiator, with the remaining conditions unchanged, as presented in Table 1 and supplementary material (Table S1). The SEC analysis of the free polymers formed in such different conditions (Fig. S2) show the decreasing of bimodality with the lower concentration of ATRP macro-initiator MCC$\mathrm{Br}$, supporting the possibility for the heterogeneity of the polymerization through the effect of the solid phase.

Thus, a thorough knowledge on the partition of the ATRP reactants among the phases involved should be used in order to design synthetic brushes with a target size. Multiphase polymer reaction engineering modeling tools, including also the plausible effect of radical termination in ATRP, are potentially helpful to design such synthesis conditions and also to discriminate the mechanisms leading to bimodality, but this subject is beyond the scope of the present paper.

SEM analysis illustrated in Fig. 7 (see also Supplementary material) shows that the native MCC particles have characteristic dimension around $20 \mu \mathrm{m}$ while the MCC- P(4VP) and MCC-PAN particles present correspondent dimensions in the order of 40 to $50 \mu \mathrm{m}$. The observed increase in particle size is a consequence of the ATRP grafting of these synthetic polymers in the native MCC.

The final goal of this research is the assessment of the hybrid MCC-P (4VP) and MCC-PAN materials to target polyphenols present in winemaking, olive oil and almond residues, namely when compared with a diverse range of alternative synthetic adsorbents. Table 2 contains a brief description of the adsorbents considered within this purpose. Besides the MCC-P(4VP) and MCC-PAN synthetized adsorbents, the native 

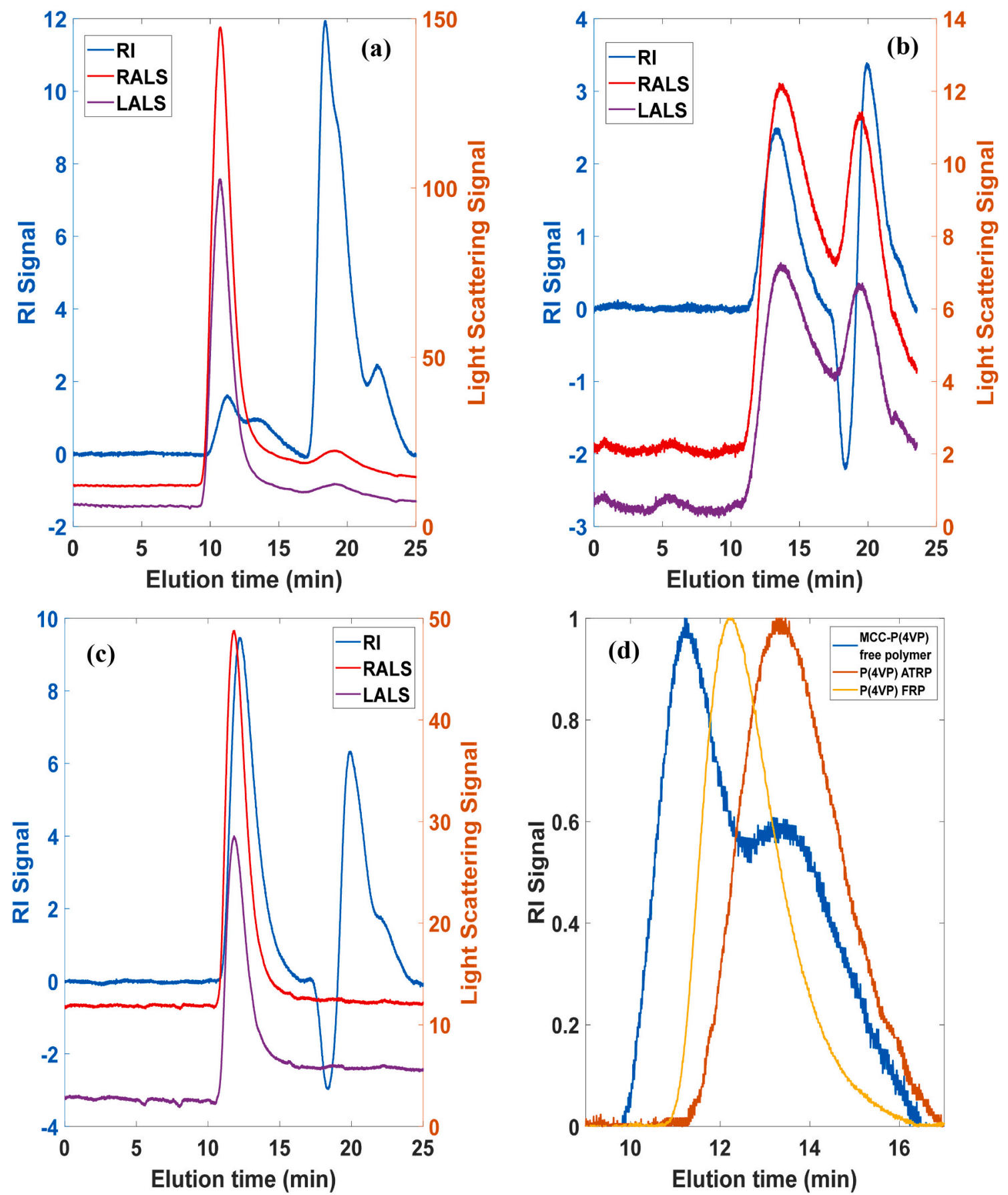

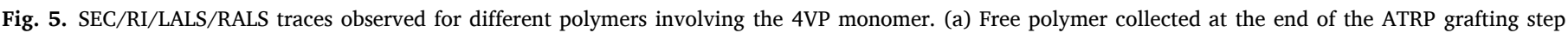

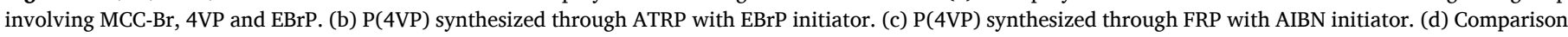
of the RI signals for these three samples. Details on the synthesis conditions of these polymers can be found in Table 1 .

MCC particles (20 $\mu \mathrm{m}$, cotton linters) and different commercial resins often used for polyphenols targeting were included in the testing runs. Moreover, 4VP and AN based adsorbents, synthesized via FRP in the framework of our previous researches [16-18], were also used with grape pomace extracts, olive mill wastewater and almond extracts/ blanching wastewater.

Fig. 8 illustrates a relevant outcome of the present work considering the targeting of the polyphenols in an EtOH/water 80/20 grape pomace extract as a case study. Indeed, we show that a high polyphenol retention is possible in the MCC-P(4VP) adsorbent (c.a. $90 \%$ measured at 280 $\mathrm{nm}$ ) while only a small fraction is adsorbed by the MCC or MCC-PAN materials (c.a. $10 \%$ at $280 \mathrm{~nm}$ ). Fig. 8 is also used to illustrate the quantification method here considered for polyphenols retention by the different adsorbents. Our calculation procedure is based on the comparison of the area of the HPLC-DAD chromatogram of the original extract with that observed for the sample collected after the loading in the adsorbent. Because the UV absorbance of the different polyphenols changes with the wavelength, fixed values for $\lambda$ were selected, namely $280,320,360$ and $380 \mathrm{~nm}$. The evaluation at $280 \mathrm{~nm}$ was mostly considered for the different adsorbents assessment, as discussed below, but this analysis can be extended to other fixed wavelength values or even considering the whole UV-vis spectra (see also the results concerning polyphenols recovery below presented). These complexities are also illustrated for the dynamics of adsorption in the Supplementary 

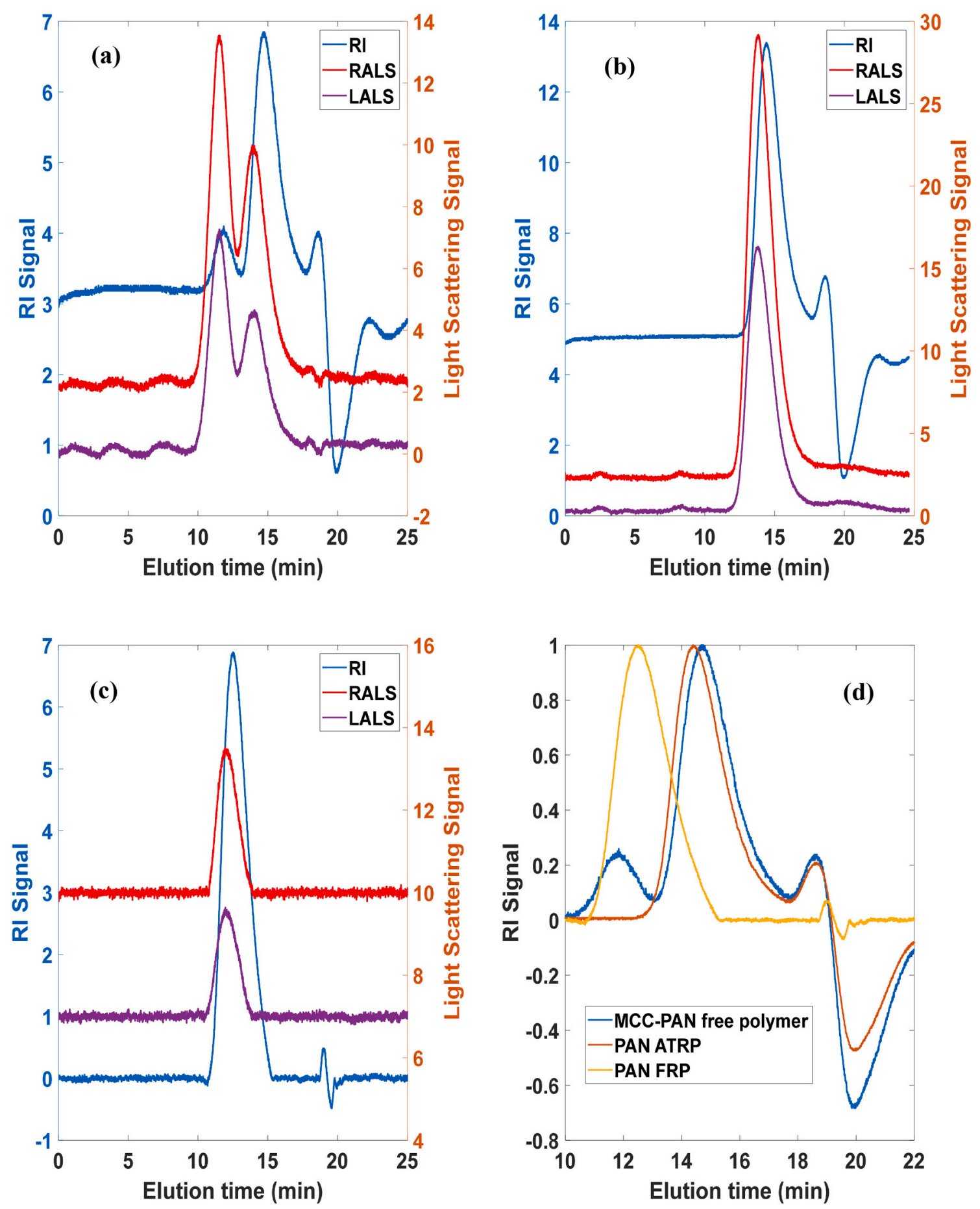

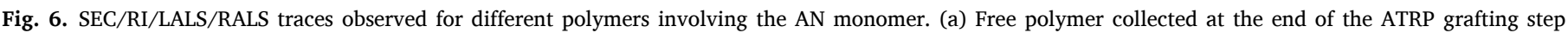

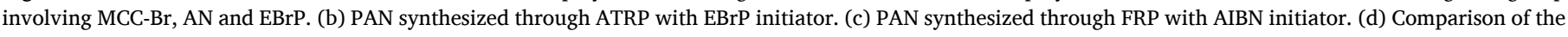
RI signals for these three samples. Details on the synthesis conditions of these polymers can be found in Table 1 .

Material (Figs. S3 to S5). Besides the plethora of varied compounds absorbing at very different wavelengths, the competitive displacement of specific molecules during the adsorption due to the effect of other species is another mechanism hardening the monitoring of such processes.

Fig. 9 presents a concise view of results for the adsorption of different grape pomace extracts with all materials here considered. These results follow from the extension to other samples of the analysis described in Fig. 8 for the specific cases EtOH/water 80/20 with MCC-P(4VP), MCC and MCC-PAN adsorbents. Results in Fig. 9 highlight the higher polyphenol retention that is achieved with the MCC-P(4VP) adsorbents comparatively not only to MCC or MCC-PAN but also to the commercial resins (DAX-8, XAD4 and XAD7HP) or other 4VP/PAN based materials (P(4VP-EGDMA) and P(4VP-AN-DVB)). Three different grape pomace extracts were considered in this analysis, namely those obtained with $\mathrm{EtOH} /$ water 80/20, ACN/DMF 50/50 and EtAc/EtOH 50/50, because the extraction solvent is an important designing parameter for real industrial applications (targeted polyphenols, process efficiency, ecological footprint, etc). Considering the average of the polyphenols retention achieved for the three grape pomace extracts, MCC-P(4VP) scores 79\% 


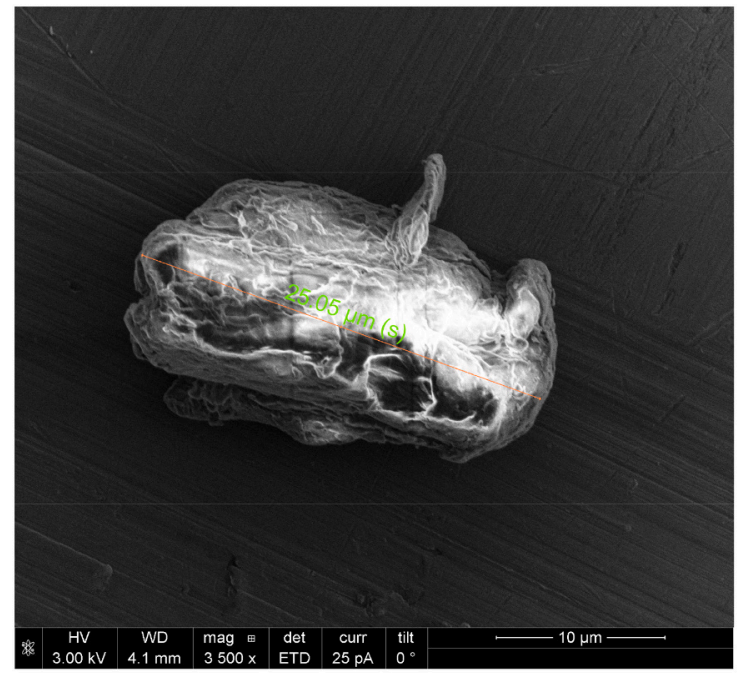

(a)

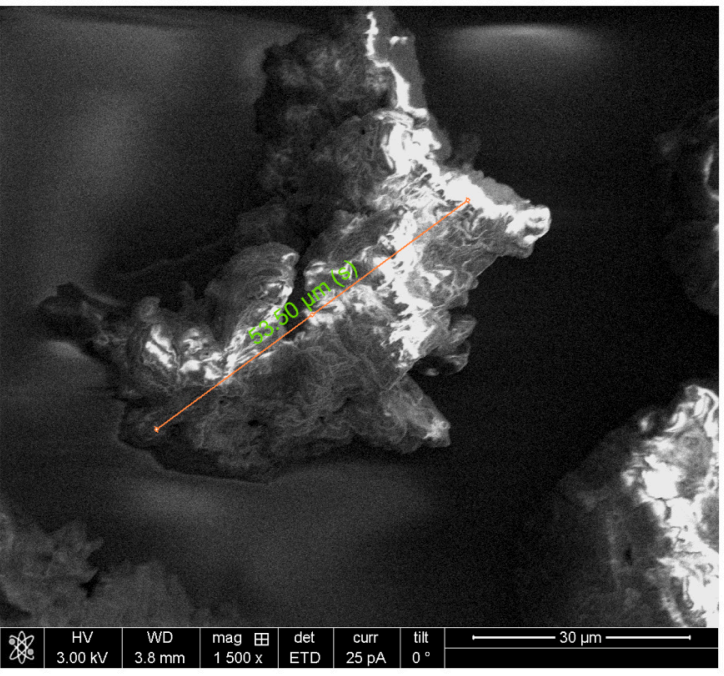

(c)

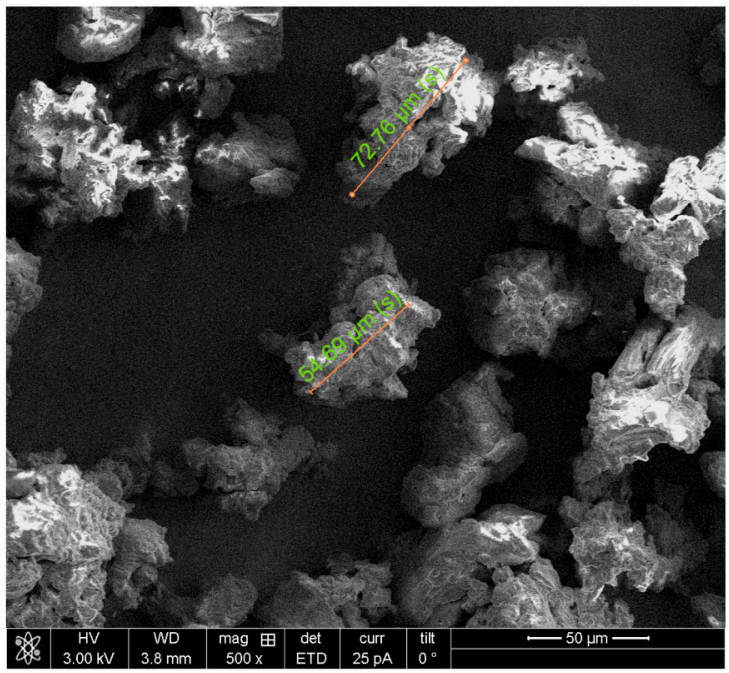

(b)

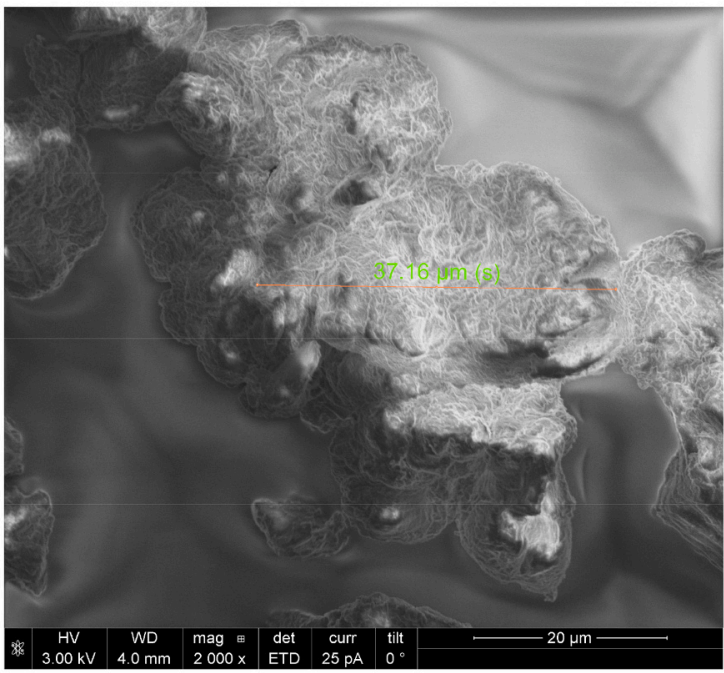

(d)

Fig. 7. SEM images showing the morphology of some particles addressed in this research. (a) Native microcrystalline cellulose particles (MCC). (b) and (c) ATRP synthesized hybrid cellulose-poly(4-vinylpyridine) particles (MCC-P(4VP)). (d) ATRP synthesized hybrid cellulose-polyacrylonitrile particles (MCC-PAN).

Table 2

Description of the different adsorbents used to target polyphenols in winemaking, olive oil and almond residues.

\begin{tabular}{|c|c|}
\hline Adsorbent & Product description \\
\hline MCC-P(4VP) & $\begin{array}{l}\text { Synthesized through BIBB immobilization in MCC followed by } \\
\text { ATRP grafting of } 4 \mathrm{VP}\end{array}$ \\
\hline MCC & Commercial microcrystalline cellulose \\
\hline MCC-PAN & $\begin{array}{l}\text { Synthesized through BIBB immobilization in MCC followed by } \\
\text { ATRP grafting of AN }\end{array}$ \\
\hline DAX-8 & $\begin{array}{l}\text { Commercial resin (Supelite }{ }^{\mathrm{TM}} \text {, polymethylmethacrylate resin of } \\
\text { moderate polarity) }\end{array}$ \\
\hline XAD4 & $\begin{array}{l}\text { Commercial resin (Amberlite }{ }^{\circledR} \text {, hydrophobic polyaromatic resin } \\
\text { based on styrene-divinylbenzene) }\end{array}$ \\
\hline XAD7HP & $\begin{array}{l}\text { Commercial resin (Amberlite }{ }^{\circledR} \text {, moderately polar acrylic ester } \\
\text { resin) }\end{array}$ \\
\hline $\begin{array}{l}\text { P(4VP- } \\
\text { EGDMA) }\end{array}$ & $\begin{array}{l}\text { Crosslinked polymer particles synthesized with 4VP/EGDMA 51/ } \\
49 \mathrm{~mol} / \mathrm{mol} \text { through free radical polymerization with AIBN as } \\
\text { initiator and ACN/DMF } 30 / 70 v / v \text { as precipitation solvent }\end{array}$ \\
\hline $\begin{array}{l}\text { P(4VP-AN- } \\
\text { DVB) }\end{array}$ & $\begin{array}{l}\text { Crosslinked polymer particles synthesized with } 4 \mathrm{VP} / \mathrm{AN} / \mathrm{DVB} 45 \text { / } \\
45 / 10 \mathrm{~mol} / \mathrm{mol} / \mathrm{mol} \text { through free radical polymerization with AIBN } \\
\text { as initiator and ethanol/water } 67 / 33 v / v \text { as precipitation solvent }\end{array}$ \\
\hline
\end{tabular}

followed by P(4VP-AN-DVB) with 72\% and P(4VP-EGDMA) with 54\% (in spite of the almost nil retention observed with the ACN/DMF 50/50 extract). Much lower retention values are observed with the commercial resins or the MCC and MCC-PAN materials (in the range 10 to $30 \%$ ).

The outcomes above described are a consequence of the specific binding capabilities associated to adsorbents based on 4VP, as detailed in ref. [7]. The $-\mathrm{N}=$ group in the pyridyl moiety contains one extra electron pair that is not involved in the ring conjugation system. This annular enclosed conjugated system is formed because each atom in the ring contains one $\mathrm{p}$ orbital and each $\mathrm{p}$ orbital adds one electron to the total of six p electrons. The bonding between the carbon and nitrogen atoms in the pyridyl group is made through $\mathrm{sp}^{2}$ hybrid orbitals and a $\mathrm{sp}^{2}$ hybrid orbital is also present in the nitrogen atom that is occupied by a lone pair of electrons [7]. Therefore, this lone pair of electrons is able to participate in strong H-bonding mechanisms, as well as to bind with protons. Indeed, the pyridyl group behaves as a proton acceptor and is also a good ligand for multiple metal ions, giving rise to complexation [7]. Due to these specific features, adsorbents based on 4VP also present a strong binding capacity for many kinds of polyphenols, as shown in our previous works [16-18]. Comparison of the results here presented with 

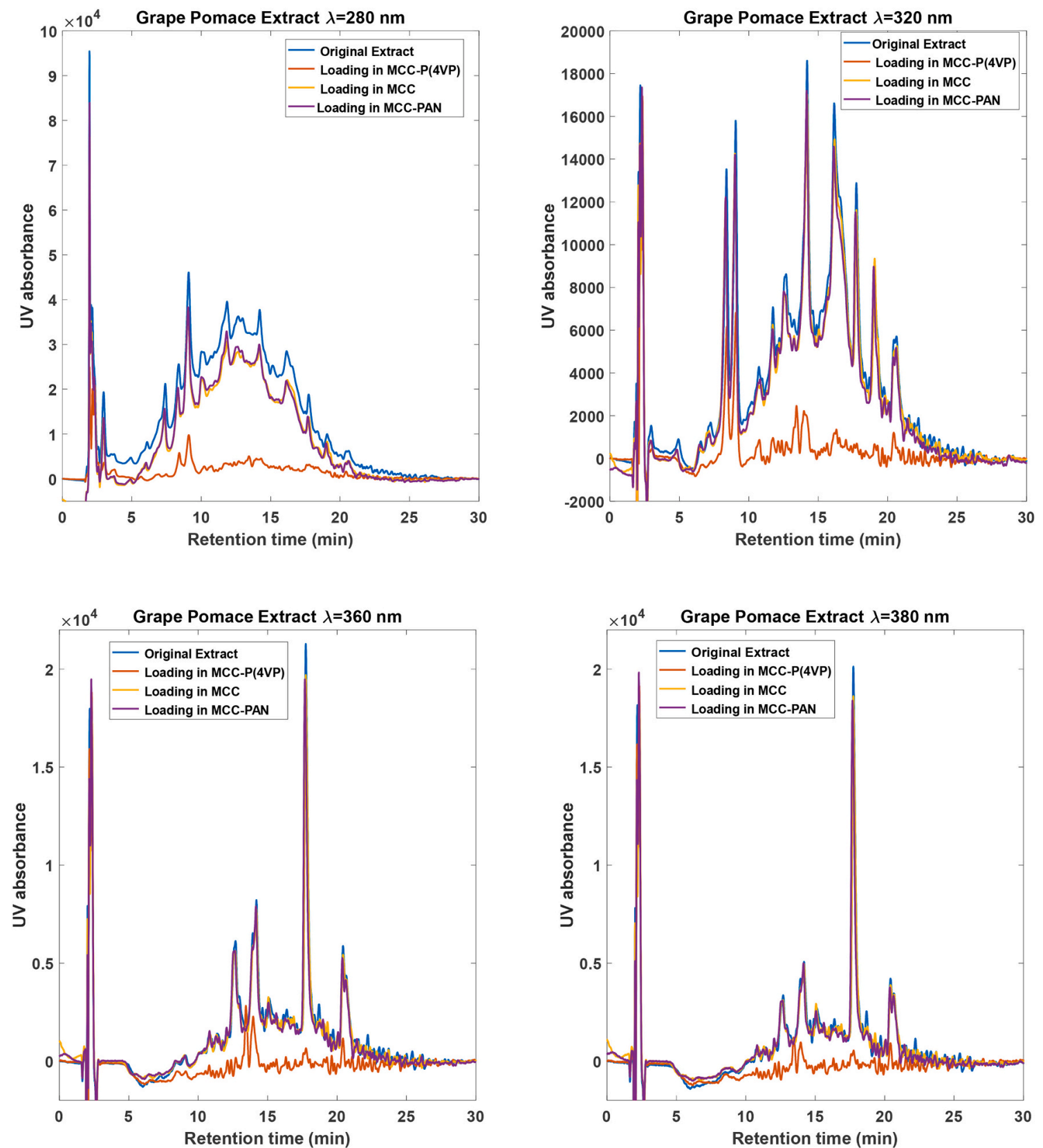

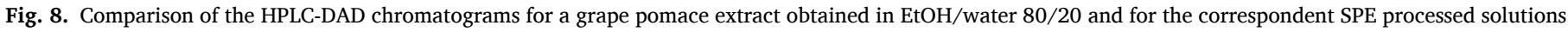

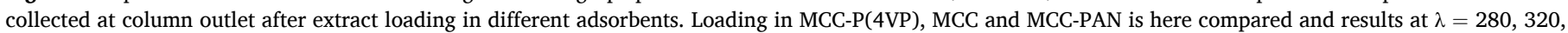
360 and $380 \mathrm{~nm}$ are presented.

MCC-P(4VP) and MCC-PAN adsorbents also confirm the stronger interaction of the pyridyl group with polyphenols comparatively to the nitrile group (the binding stabilization provided by the conjugated system in the pyridyl moiety plays as important role). Note that the $\mathrm{N}_{2}$ adsorption/ desorption results put in evidence a very low specific surface area and pore volume, not only for the native MCC, but also for the hybrid MCC-P (4VP) and MCC-PAN particles synthesized (see Table S2). These outcomes corroborate that the high polyphenol retention observed with the hybrid MCC-P(4VP) particles is mostly due to the functionalization of the external surface with the pyridyl functional groups.

Results for the adsorption of hull and shell almond polyphenols contained in EtOH/water 80/20, ACN/DMF 50/50 and EtAc/EtOH 50/ 50 are presented in Fig. 10. These measurements confirm the superior capacity of the MCC-P(4VP) adsorbent to retain polyphenols contained in almond residues also when solvents with high organic content are used. The strong interaction of the pyridyl group with many polyphenols, as above discussed, gives support to these outcomes. The effect of hydrophobic interactions, which is the driving force for the adsorption of polyphenols in many adsorbents used with plant extracts, has a lower impact in retention when working with 4VP based materials. Among all adsorbents tested, taking the average retention for the four almond extracts considered, MCC-P(4VP) scores with 76\%, followed by P(4VP-AN-DVB) with 63\% and P(4VP-EGDMA) with 55\%. Again, a very small retention is observed with the commercial resins or with MCC and MCC-PAN materials.

In Fig. 11 are presented the retained fractions measured with aqueous extracts containing polyphenols. Olive mill wastewater and almond blanching wastewater were processed with the different adsorbents and the results show an important contribution of hydrophobic interactions for adsorption. Commercial resins are now able to retain 


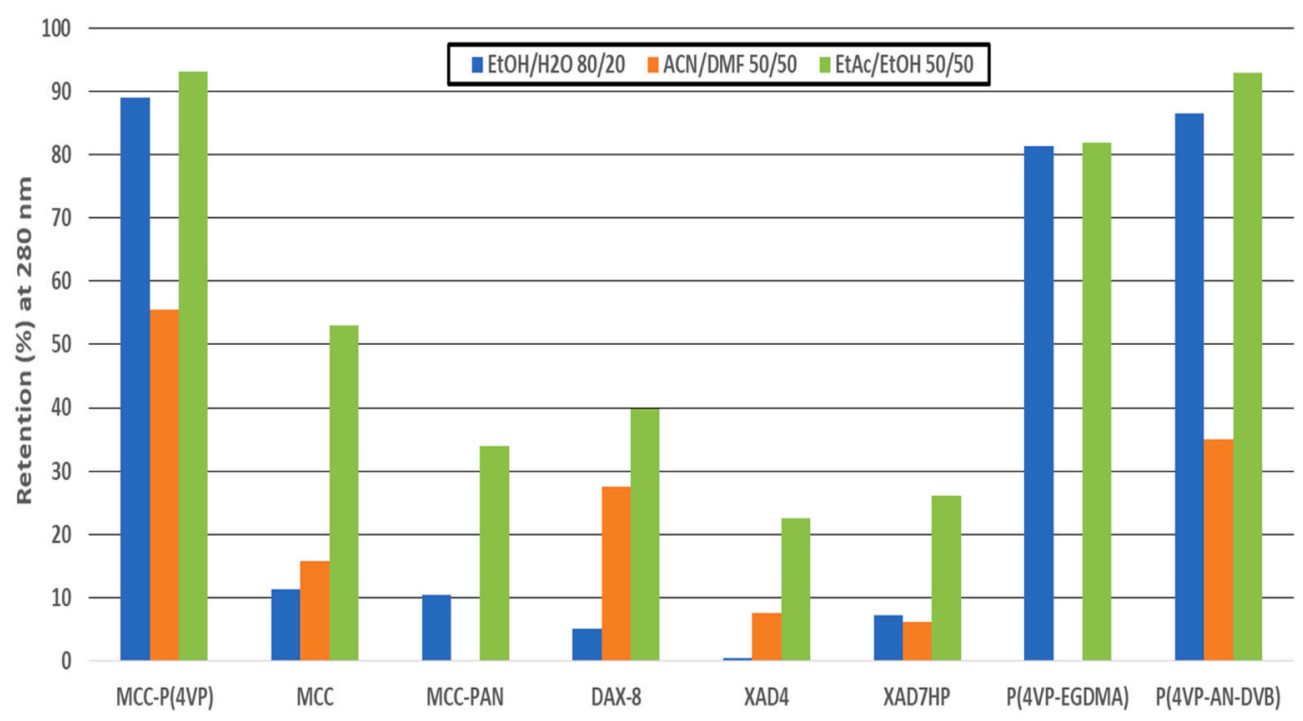

Fig. 9. Comparison for the retention in different adsorbents observed with different grape pomace extracts (obtained with EtOH/water 80/20, ACN/DMF 50/50 and EtAc/EtOH 50/50 as solvents). Measurements were performed in SPE columns and retention was calculated using UV absorbance at $\lambda=280 \mathrm{~nm}$.

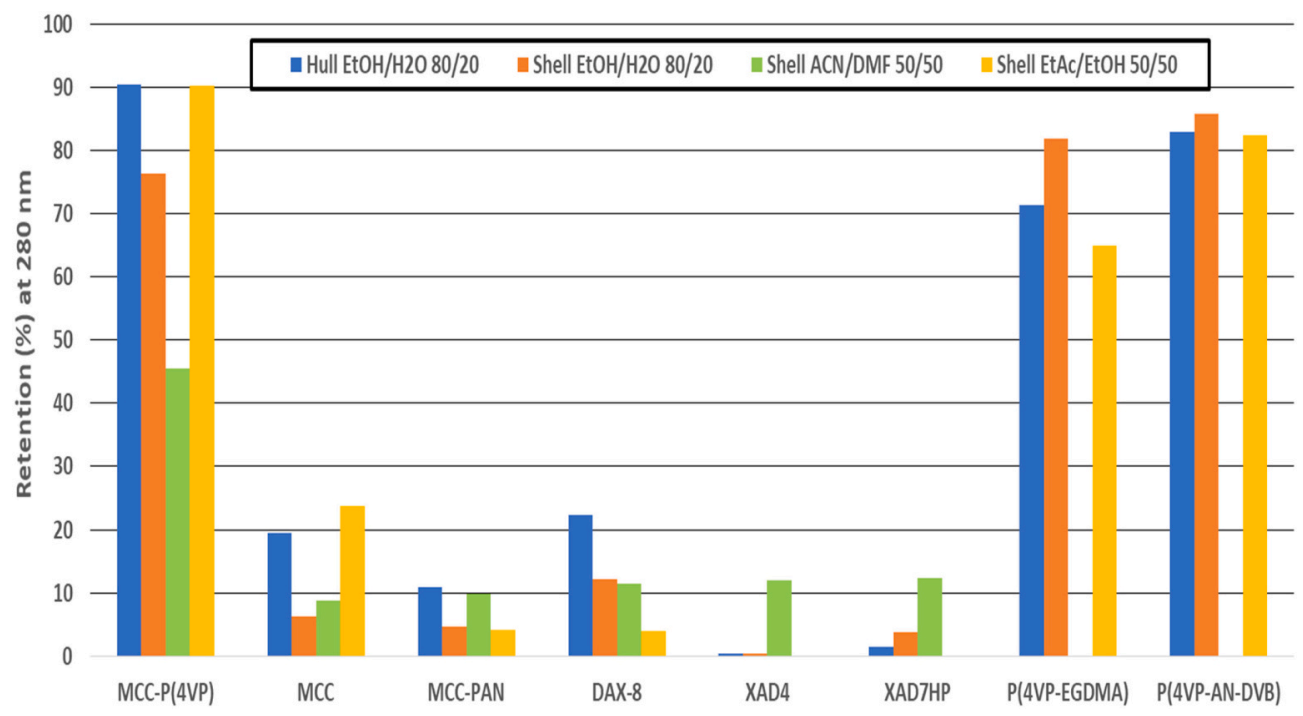

Fig. 10. Comparison for the retention in different adsorbents observed with different almond hull and almond shell extracts (obtained with EtOH/water $80 / 20$, ACN/DMF 50/50 and EtAc/EtOH 50/50 as solvents). Measurements were performed in SPE columns and retention was calculated using UV absorbance at $\lambda=$ $280 \mathrm{~nm}$.

appreciable amounts of polyphenols (e.g. DAX-8 scores $74 \%$ for the average of the two extracts) but the best outcomes are observed with the two 4VP based adsorbents synthetized via FRP (89\% for P(4VP-AN-DVB) and $83 \%$ for P(4VP-EGDMA)). The adsorbent MCC-P(4VP) scores now with $44 \%$ average retention and lower values are observed for MCC (13\%) or MCC-PAN (21\%). The combined effect between the adsorption capabilities of the pyridyl moieties and the hydrophobic interactions enhanced by the crosslinkers (EGDMA and DVB) reason the high polyphenol retention observed with the P(4VP-EGDMA) and P(4VP-ANDVB) adsorbents. On the other hand, the $\mathrm{pH}$ conditions of the olive mill wastewater $(\mathrm{pH}=5)$ and of the almond blanching wastewater $(\mathrm{pH}$ $=6.1$ ) also contribute for their relatively lower adsorption capability as measured with the MCC-P(4VP) material (note the high P(4VP) content of this adsorbent). Indeed, as demonstrated before with $\mathrm{P}(4 \mathrm{VP})$ based resins for heavy metals adsorption, the $\mathrm{pH}$ has an important effect on amounts and selectivity of the different cations retained [7]. When the $\mathrm{pH}$ is low enough, the pyridine ring becomes protonated and positively charged molecules cannot be adsorbed whereas anions can be retained by electrostatic interactions. Similar pH effects are possible for phenolic acids in olive mill wastewater and almond blanching wastewater, namely by shifting the equilibrium for phenolic acids dissociation and $\mathrm{P}$ (4VP) protonation. However, the most important factor hindering polyphenols retention at acid conditions with MCC-P(4VP) materials should be the decrease of the pyridyl group capacity to establish hydrogen bonding with these compounds as a consequence of protonation. Adjustment of $\mathrm{pH}$ is therefore a tool that can be considered to increase adsorption rates with MCC-P(4VP) materials but our option here was the use of the simple processing conditions concerning the direct processing of the aqueous extracts.

Overall, considering the nine different extracts described in Figs. 9-11, the MCC-P(4VP) adsorbent presents an average retention capability of $70 \%$, a slightly higher value $(72 \%)$ results for $\mathrm{P}(4 \mathrm{VP}-\mathrm{AN}$ DVB), while $61 \%$ stands for P(4VP-EGDMA). These results confirm the good retention capability of 4VP based adsorbents for polyphenols considering a broad range of working conditions (much lower retention averages are observed with the other adsorbents). When only the 


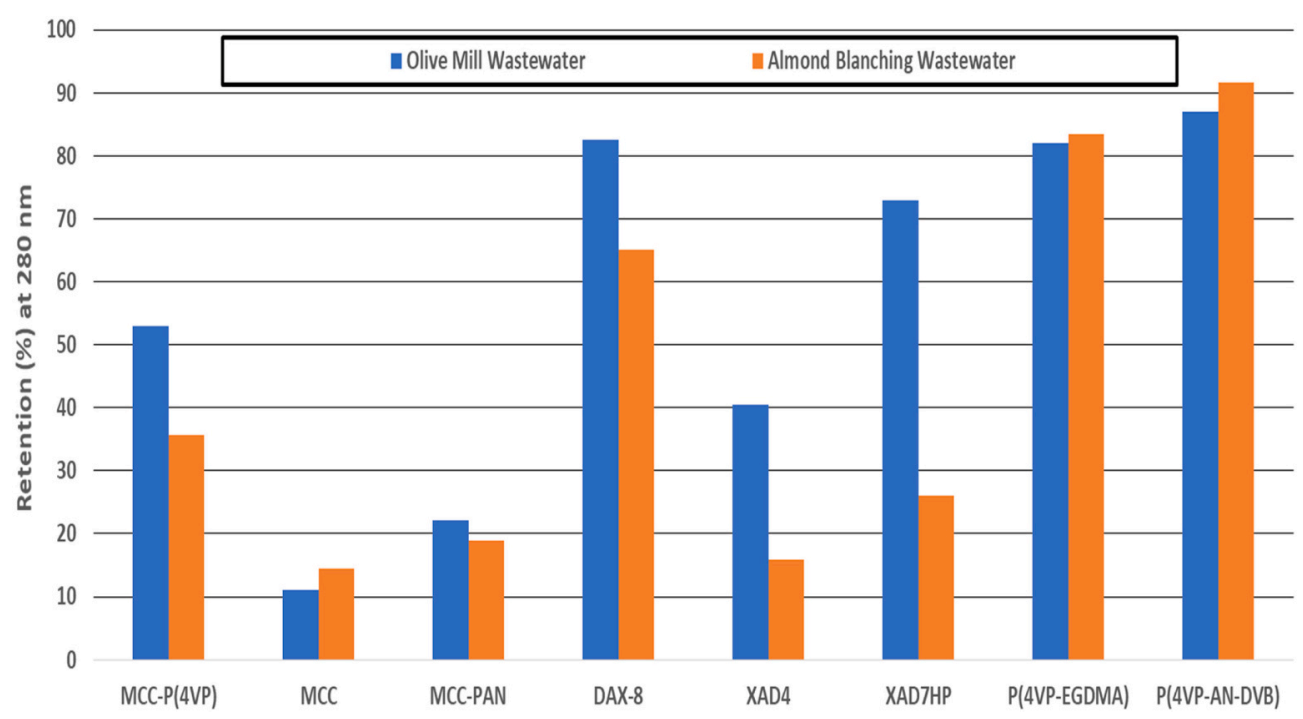

Fig. 11. Comparison for the retention in different adsorbents observed with olive mill wastewater and almond blanching wastewater. Measurements were performed in SPE columns and retention was calculated using UV absorbance at $\lambda=280 \mathrm{~nm}$.

organic or low water content extracts are considered (decreasing the hydrophobic effects and therefore enhancing adsorbent specificity), the MCC-P(4VP) scores with 77\% while P(4VP-AN-DVB) and (4VP-EGDMA) with $67 \%$ and $54 \%$, respectively.

Note that, as stated above, with complex mixtures like plant extracts, the polyphenols are adsorbed differently depending particular interactions with the polymer network moieties. Thus, when the simple global polyphenol retention is assessed, the value changes with the wavelength due to particular spectra of the species retained. This effect is highlighted in Fig. 12 by comparing the polyphenol retention measured at 280 and $380 \mathrm{~nm}$ for almond shell and grape pomace extracts in ACN/DMF 50/50 and considering the different adsorbents. These results also demonstrate the higher capacity of the MCC-P(4VP) adsorbent to retain polyphenols even when stronger organic solvents (ACN/DMF 50/50) are used. A more comprehensive view of polyphenols adsorption/desorption change with wavelength can be observed in Fig. 13 (see also Supplementary Material).

Results above presented demonstrate the good capability of the MCC-P(4VP) adsorbents to retain polyphenols in different plant extracts.
This is an important issue for industrial processes dealing with very high liquid volumes containing polyphenols at low concentration (e.g. food industry). In practice, the transport of such high volumes for secondary processing must be avoided and therefore efficient enrichment mechanisms should be considered. In the simplest case, this enrichment is just a volume reduction [22] and adsorbents with high retention capability play an important role in this context. These features were above illustrated with the 4VP-based materials synthesized in our research.

Additionally, besides the concentration (enrichment) process, the recovery and fractionation of the polyphenols is also sought in many industrial applications. These purification and fractionation applications require the assessment of polyphenols desorption after their loading in the used materials. Selective desorption processes are often triggered by the use of a sequence of solvents with different elution strengths and/or temperature-swing mechanisms. The application of the MCC-P(4VP) adsorbents in the purification and fractionation of polyphenols in grape pomace, almond residues and olive mill wastewater was also addressed in the present work as illustrated in Fig. 13 (see also Supplementary Material).

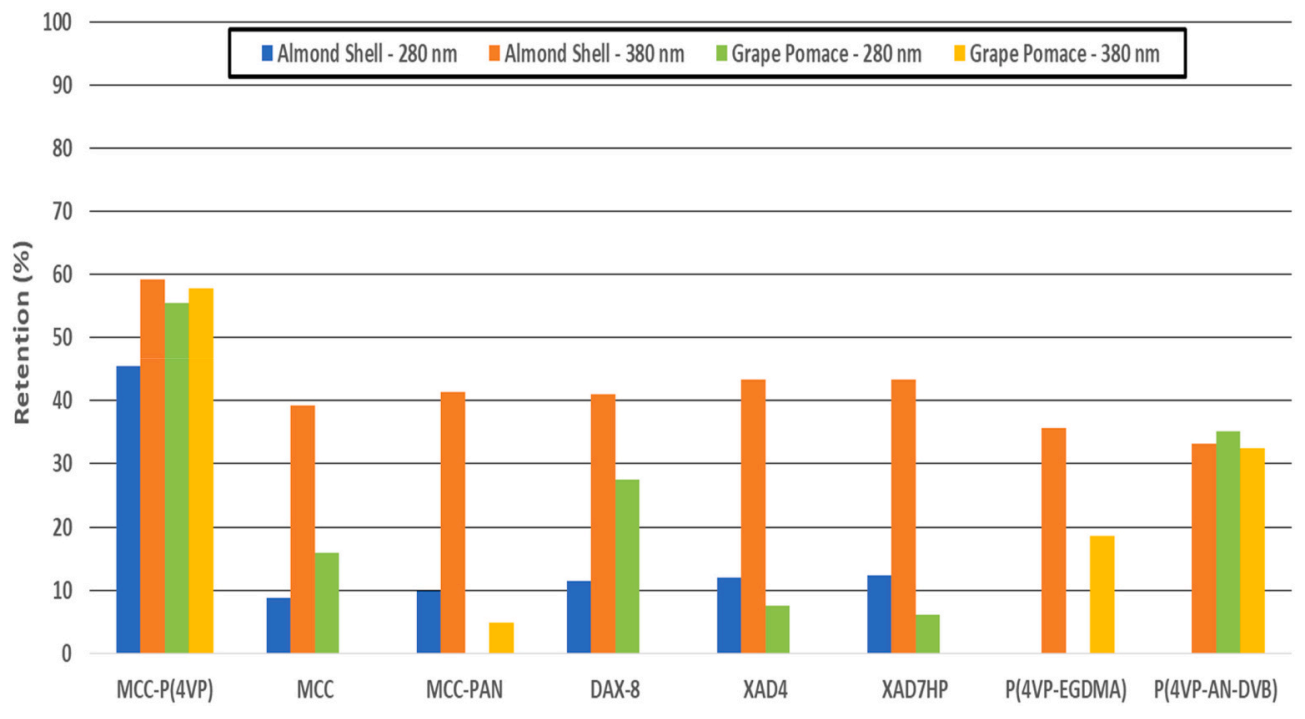

Fig. 12. Comparison for the retention measured at $\lambda=280 \mathrm{~nm}$ and $\lambda=380 \mathrm{~nm}$ considering different adsorbents with almond shell and grape pomace extracts obtained in ACN/DMF 50/50. 

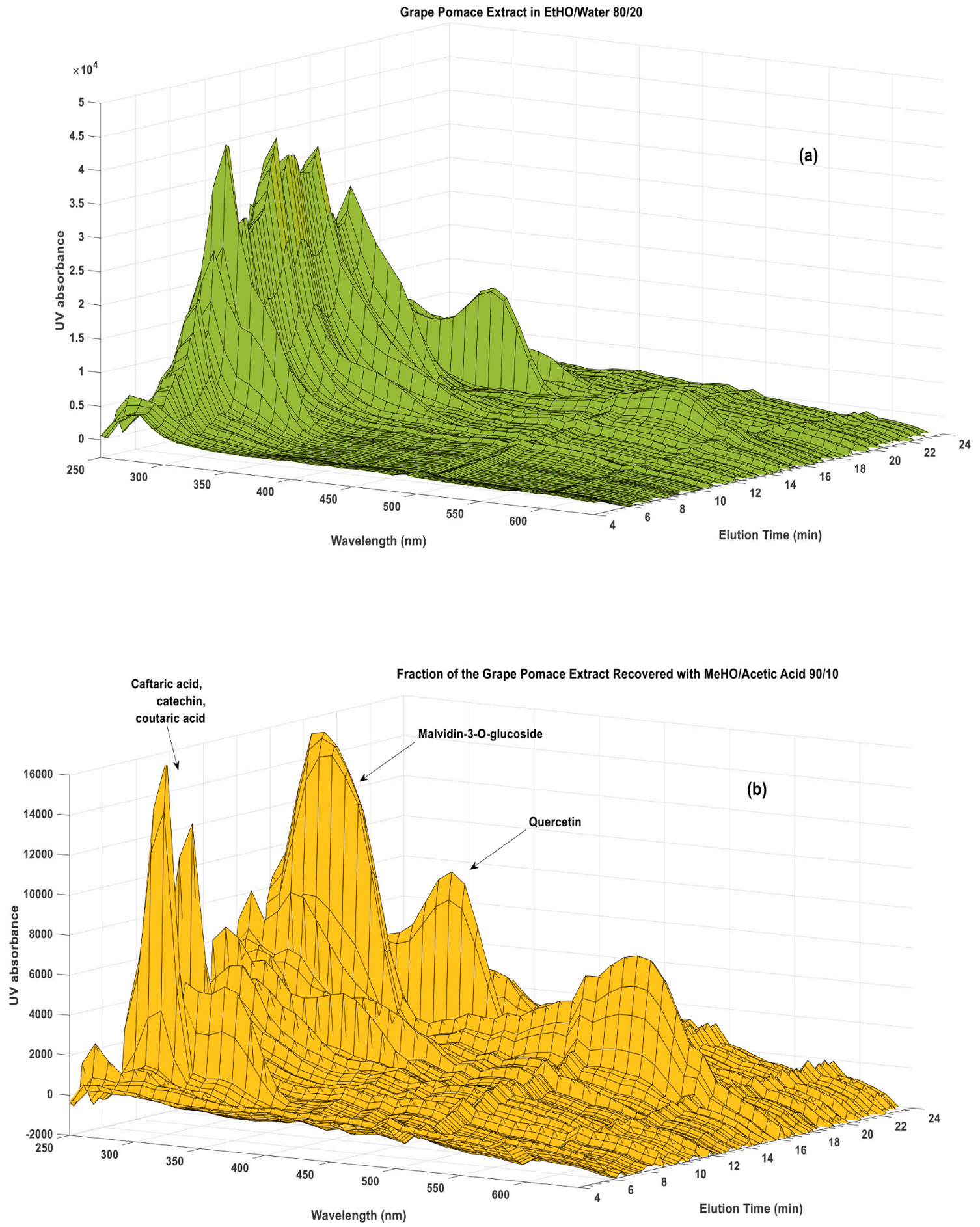

Fig. 13. HPLC-DAD analysis of: (a) a grape pomace extract obtained in EtOH/water $80 / 20$ and (b) a fraction of this extract recovered in MeOH/acetic acid $90 / 10$ after loading the grape pomace extract with the MCC-P(4VP) adsorbent.

Fig. 13(a) shows the HPLC-DAD chromatogram observed for a grape pomace extract obtained in EtOH/Water 80/20 and reveals a complex mixture of phenolic compounds that are known to be present in wine and vinery residues. Citric acid, caftaric acid, catechin, $p$-coutaric acid, quercetin glucuronide, resveratrol, quercetin and kaempferol are just a few examples of polyphenols present in such kinds of extracts $[17,23-25]$, besides different sorts of anthocyanins and their 3-O-monoglucosides [23]. On the other hand, Fig. 13(b) shows a fraction recovered after the loading of this grape pomace extract in the MCC-P (4VP) adsorbent. This specific fraction was recovered with $\mathrm{MeOH} /$
Acetic acid 90/10 (see Section 2.8) and the HPLC-DAD analysis presented in Fig. 13(b) highlights the purification and fractionation achieved with this simple sorption/desorption procedure. Indeed, the comparison of Fig. 13(a)-(b) shows the production of fraction enriched with the malvidin-3-O-glucoside anthocyanin, as indicated by the increase of absorption intensity in the visible region of the spectrum. A set of well-defined peaks correspondent to other polyphenols are also now discernible in HPLC-DAD analysis as consequence of this simple sorption/desorption process. If needed, further sorption/desorption steps should be considered to achieve higher polyphenol purification/ 
fractionation, depending on the application requirements.

A similar analysis for an almond shell extract obtained in EtOH/ water $80 / 20$ and handled through a sorption/desorption process with the MCC-P(4VP) adsorbent is presented in the Supplementary Material. The initial extract exhibits a complex mixture of polyphenols including catechin, procatechuic acid, epicatechin, vanillic acid, quercetin and quercetin-glucosides, kaempferol and kaempferol-glucosides, as well as isorhamnetin and isorhamnetin glucosides, among others [22,26,27]. Comparison of initial/processed extract demonstrates the purification of the almond shell extract achieved with this simple sorption/desorption process. The fraction recovered was enriched in isorhamnetin-3-O-rutinoside and also in quercetin-glucosides (different isomers, diglucosides or galactosides are possible $[22,26,27])$. These outcomes are useful for downstream processing of almond residues and also for analytic purposes, namely for sample preparation aiming at polyphenols identification and quantification.

A first application of the MCC-P(4VP) adsorbent with aqueous extracts is presented in Appendix. Polyphenols present in almond blanching wastewater were adsorbed in the MCC-P(4VP) material and a fraction afterwards recovered with $\mathrm{MeOH} /$ Acetic acid 90/10. The purification of the initial almond blanching wastewater was achieved and an isorhamnetin-3-O-rutinoside rich fraction was obtained. Note also the difference between the initial phenolic profile for the almond shell extract in EtOH/water 80/20 and almond blanching wastewater (see Appendix) caused by the raw materials used and mainly by the extraction solvents (EtOH/water 80/20 and water). In both cases the MCC-P (4VP) adsorbent was usefully used for purification/fractionation of the contained polyphenols.

The processing of olive mill wastewater with the MCC-P(4VP) adsorbent is illustrated in the Supplementary Material. The main polyphenols in this kind of complex aqueous extracts belong to the classes of phenolic alcohols (e.g. tyrosol and hydroxytyrosol), phenolic acids (vanillic acid, caffeic acid, sinapic, vanillin acid, etc), secoiridoids and derivatives (oleuropein, elenolic acid, oleoside, etc) and flavonoids (apigenin, luteolin, luteolin-7-glucoside, etc) [28-30]. In Appendix is also presented the phenolic profile for a fraction recovered with $\mathrm{MeOH} /$ water $(\mathrm{pH}=2) 90 / 10$ after the loading of the olive mill wastewater in MCC-P(4VP). The fraction thus recovered was enriched in secoiridoids and flavonoids while other major components of the olive mill wastewater, namely many phenolic alcohols and acids, were successfully removed from the solution using this simple sorption/desorption process with the MCC-P(4VP) materials. These results show that the use of the adsorbents here developed deserves technological scrutiny for olive mill wastewater valorization, namely in integrated processes including crystallization [31] or membranes filtration [32].

\section{Conclusion}

Microcrystalline cellulose particles were grafted via ATRP with a high content of poly(4-vinylpyridine) brushes. The hybrid particles were used as adsorbents to target polyphenols in different kinds of plant extracts derived from grape pomace, almond residues and almond processing wastewater, as well as olive oil production effluents. It was observed that the morphology of the final hybrid particles keeps appropriated for use in continuous sorption/desorption processes. For comparison purposes, a hybrid adsorbent resulting from the ATRP grafting of polyacrylonitrile in cellulose was also synthesized.

The produced hybrid adsorbents were assessed for the retention of polyphenols in organic solvents (ACN/DMF 50/50 and EtAc/EtOH 50/ 50), organic/aqueous mixtures with low water content (EtOH/water 80/ 20) and completely aqueous extracts (almond and olive mill wastewater). These assays included also the use of other adsorbents, namely the native microcrystalline cellulose, commercial resins (DAX-8, XAD4 and XAD7HP) as well as other polymer networks synthesized via radical polymerization with 4-vinylpyridine as co-monomer. Considering the average polyphenol retention as comparative parameter and nine different extracts, the MCC-P(4VP) adsorbent and the 4VP based networks present a much higher performance (c.a. 70\% retention) comparatively to MCC, MCC-PAN or commercial resins (retention in the range of 10 to $20 \%$ observed for many materials). Averaging with exclusion of the aqueous extracts leads to an even higher retention capability for MCC-P(4VP) (c.a. 77\%) also when compared with the other 4VP based networks. These results are grounded by the strong binding capacity of the pyridyl group towards many polyphenols and show that the design of adsorbents working with a lower impact of the hydrophobic interactions is achieved increasing the 4VP content in the materials.

MCC-P(4VP) adsorbents were also used for purification and fractionation of polyphenols using simple sorption/desorption steps. With this straightforward approach, a fraction enriched with the malvidin-3$O$-glucoside anthocyanin was obtained from a grape pomace extract; fractions containing high content of isorhamnetin-3-O-rutinoside were isolated from almond shell as well as from almond blanching wastewater and fractions enriched in secoiridoids and flavonoids were produced from olive mill wastewater while many phenolic alcohols and acids were removed. These results show the potentiality of the MCC-P(4VP) adsorbents to improve downstream processing of polyphenols and increase the biorefining efficiency (e.g. allowing direct handling of organic extracts with high polyphenol concentration).

This research also shows that the use of controlled radical polymerization techniques, namely ATRP, offers many possibilities for the designing of natural-synthetic functional materials to be used as tailored adsorbents. The combination of molecular imprinting with ATRP functional grafting in cellulose to gain materials with molecular recognition features and the optimization of the cellulose/synthetic incorporation ratios in the final materials are issues to be addressed in future works. A comprehensive cost-benefit analysis on the use of the kind of adsorbents here addressed is also needed including sustainability factors as well as the potential increase on process efficiency and flexibility due to the use of tailored materials. The formation of a polymer population not following the expected homogeneous process here demonstrated through SEC analysis also deserves scrutiny in future works to gain precision in the designing of synthetic brushes with a target size. This is particularly important when monomers with strong H-bond capability, such as $4 \mathrm{VP}$, are involved.

\section{Declaration of Competing Interest}

The authors declare that they have no known competing financial interests or personal relationships that could have appeared to influence the work reported in this paper.

\section{Acknowledgements}

Catarina Gomes and Rolando Dias are grateful to the Foundation for Science and Technology (FCT, Portugal) for financial support by national funds FCT/MCTES to CIMO (UIDB/00690/2020). We acknowledge to Rute Gaspar (Torre de Moncorvo) the donation of almond fruits for this research. The contribution of the Chemical Engineering master students of IPB Cristina Nogueira and Cláudia Martins is also acknowledged.

Mário Rui Costa also acknowledges financial support by FCT through Base Funding - UIDB/50020/2020 of the Associate Laboratory LSRELCM - funded by national funds through FCT/MCTES (PIDDAC).

We also acknowledge the contribution of the Professor Agostinho Almeida group with the bromine determinations by ICP-MS at LAQV/ REQUIMTE-FFUP.

This article is also a result of the project "BacchusTech - Integrated Approach for the Valorisation of Winemaking Residues" (POCI-01-0247FEDER-069583), supported by the Competitiveness and Internationalization Operational Programme (COMPETE 2020), under the PORTUGAL 2020 Partnership Agreement, through the European 
Regional Development Fund (ERDF).

All the authors certify that they have participated sufficiently in the work to take public responsibility for the content, including participation in the concept, design, analysis, writing, or revision of the manuscript.

\section{Appendix A. Supplementary data}

Supplementary data to this article can be found online at https://doi. org/10.1016/j.reactfunctpolym.2021.104930.

\section{References}

[1] S. Keshipour, A. Maleki, Modification of cellulose, in: M. Mondal (Ed.), CelluloseBased Superabsorbent Hydrogels. Polymers and Polymeric Composites: A Reference Series, Springer, Switzerland, 2019, https://doi.org/10.1007/978-3319-77830-3_17.

[2] R. Kumar, R. Sharma, Synthesis and characterization of cellulose based adsorbents for removal of $\mathrm{Ni}(\mathrm{II}), \mathrm{cu}(\mathrm{II})$ and $\mathrm{Pb}(\mathrm{II})$ ions from aqueous solutions, React. Funct. Polym. 140 (2019) 82-92.

[3] S. Sethia, B.S. Kaithb, V. Kumar Saruchic, Fabrication and characterization of microwave assisted carboxymethyl cellulose-gelatin silver nanoparticles imbibed hydrogel: its evaluation as dye degradation, React. Funct. Polym. 142 (2019) $134-146$.

[4] J. Zhao, K. Zheng, J. Nan, C. Tang, Y. Chen, Y. Hu, Synthesis and characterization of lignosulfonate-graft-poly (acrylic acid)/hydroxyethyl cellulose semi interpenetrating hydrogels, React. Funct. Polym. 115 (2017) 28-35.

[5] X. Sui, J. Yuan, M. Zhou, J. Zhang, H. Yang, W. Yuan, Y. Wei, C. Pan, Synthesis of Cellulose-graft-Poly(N,N-dimethylamino-2-ethyl methacrylate) copolymers via homogeneous ATRP and their aggregates in aqueous media, Biomacromolecules 9 (2008) 2615-2620.

[6] K. Shibata, M. Hayashi, Y. Inay, Experimental and theoretical investigation of intrinsic pyridine isomer effects on physical property tuning of Metallo supramolecular polymers bearing multiple pyridine ligands, ACS Appl. Polym. Mater. 6 (2020) 2327-2337.

[7] Y. Chen, W. Zhao, J. Zhang, Preparation of 4-vinylpyridine (4VP) resin and its adsorption performance for heavy metal ions, RSC Adv. 7 (2017) 4226-4236.

[8] E.S. Yanovska, L.O. Vretik, O.A. Nikolaeva, Y. Polonska, D. Sternik, O. Yu. Kichkiruk, Synthesis and adsorption properties of 4-Vinylpyridine and styrene copolymer in situ immobilized on silica surface, Nanoscale Res. Lett. 12 (2017) 217.

[9] C. Xiao, J. Lin, Efficient removal of $\mathrm{Cr}(\mathrm{VI})$ ions by a novel magnetic 4-vinyl pyridine grafted Ni3Si2O5(OH)4 multiwalled nanotube, ACS Omega 5 (2020) 23099-23110.

[10] Y.A.B. Neolaka, Y. Lawa, J.N. Naat, A.A.P. Riwu, H. Darmokoesoemo, G. Supriyanto, C.I. Holdsworth, A.N. Amenaghawon, H.S. Kusuma, A Cr(VI)imprinted-poly(4-VP-co-EGDMA) sorbent prepared using precipitation polymerization and its application for selective adsorptive removal and solid phase extraction of $\mathrm{Cr}(\mathrm{VI})$ ions from electroplating industrial wastewater, React. Funct. Polym. 147 (2020) 104451.

[11] N. Amaly, Y. Ma, A.Y. El-Moghazy, G. Sun, Copper complex formed with pyridine rings grafted on cellulose nanofibrous membranes for highly efficient lysozyme adsorption, Sep. Purif. Technol. 250 (2020) 117086.

[12] M. Atif, C. Chen, M. Irfan, F. Mumtaz, K. He, M. Zhang, L. Chen, Y. Wang, Poly(2methyl-2-oxazoline) and poly(4-vinyl pyridine) based mixed brushes with switchable ability toward protein adsorption, separation and purification, Eur. Polym. J. 120 (2019) 109199.

[13] N. Sahiner, O. Ozay, Highly charged p(4-vinylpyridine-co-vinylimidazole) particles for versatile applications: biomedical, catalysis and environmental, React. Funct. Polym. 71 (2011) 607-615.
[14] A. Carlmark, E.E. Malmström, ATRP grafting from cellulose Fibers to create blockcopolymer grafts, Biomacromolecules 4 (2003) 1740-1745.

[15] Z. Zhang, X. Wang, K.C. Tam, G. Sèbe, A comparative study on grafting polymers from cellulose nanocrystals via surface-initiated atom transfer radical polymerization (ATRP) and activator re-generated by electron transfer ATRP, Carbohydr. Polym. 205 (2019) 322-329.

[16] C.P. Gomes, G. Sadoyan, R.C.S. Dias, M.R.P.F.N. Costa, Development of molecularly imprinted polymers to target polyphenols present in plant extracts, Processes 5 (2017) 72.

[17] C.P. Gomes, R.C.S. Dias, M.R.P.F.N. Costa, Preparation of molecularly imprinted adsorbents with improved retention capability of polyphenols and their application in continuous separation processes, Chromatographia 82 (2019) 893-916.

[18] C.P. Gomes, V. Franco, R.C.S. Dias, M.R.P.F.N. Costa, Processing of onion skin extracts with quercetin-molecularly imprinted adsorbents working at a wide range of water content, Chromatographia 83 (2020) 1539-1551.

[19] D. Oliveira, C.P. Gomes, R.C.S. Dias, M.R.P.F.N. Costa, Molecular imprinting of 5fluorouracil in particles with surface RAFT grafted functional brushes, React. Funct. Polym. 107 (2016) 35-45.

[20] R.C.S. Dias, M.R.P.F.N. Costa, Calculation of CLD using population balance equations of generating functions: linear and non-linear ideal controlled radical polymerization, Macromol. Theory Simul. 19 (2010) 323-341.

[21] C.P. Gomes, R.C.S. Dias, M.R.P.F.N. Costa, Polymer reaction engineering tools to tailor smart and superabsorbent hydrogels, in: M. Mondal (Ed.), Cellulose-Based Superabsorbent Hydrogels. Polymers and Polymeric Composites: A Reference Series, Springer, Switzerland, 2019, https://doi.org/10.1007/978-3-319-77830-3_ 19.

[22] V. Hellwig, J. Gasser, Polyphenols from waste streams of food industry: valorisation of blanch water from marzipan production, Phytochem. Rev. (2020), https://doi.org/10.1007/s11101-020-09663-y.

[23] F. He, Na-Na Liang, L. Mu, Qiu-Hong Pan, J. Wang, M.J. Reeves, Chang-Qing Duan, Anthocyanins and their variation in red wines I. Monomeric anthocyanins and their color expression, Molecules 17 (2012) 1571-1601.

[24] O. Aizpurua-Olaizola, M. Ormazabal, A. Vallejo, M. Olivares, P. Navarro, N. Etxebarria, A. Usobiaga, Optimization of supercritical fluid consecutive extractions of fatty acids and polyphenols from Vitis Vinifera grape wastes, J. Food Sci. 80 (2015) E101-E107.

[25] A.M.A. Borbala, L. Zorro, D.A. Guillen, C.G. Barroso, Study of the polyphenol content of red and white grape varieties by liquid chromatography-mass spectrometry and its relationship to antioxidant power, J. Chromatogr. A 1012 (2003) 31-38.

[26] P.E. Milbury, C.-Y. Chen, G.G. Dolnikowski, J.B. Blumberg, Determination of flavonoids and Phenolics and their distribution in almonds, J. Agric. Food Chem. 54 (2006) 5027-5033.

[27] A. Smeriglio, G. Mandalaria, C. Bisignano, A. Filocamo, D. Barreca, E. Bellocco, D. Trombetta, Polyphenolic content and biological properties of Avola almond (Prunus dulcis mill. D.a. Webb) skin and its industrial byproducts, Ind. Crop. Prod. 83 (2016) 283-293.

[28] A. Alfano, L. Corsuto, R. Finamori, M. Savarese, F. Ferrara, S. Falco, G. Santabarbara, M. De Rosa, C. Schiraldi, Valorization of olive mill wastewater by membrane processes to recover natural antioxidant compounds for cosmeceutical and nutraceutical applications or functional foods, Antioxidants 7 (2018) 72.

[29] K. De La Torre-Carbot, O. Jauregui, E. Gimeno, A.I. Castellote, R.M. LamuelaRaventós, M.C. López-Sabater, Characterization and quantification of phenolic compounds in olive oils by solid-phase extraction, HPLC-DAD, and HPLC-MS/MS, J. Agric. Food Chem. 53 (2005) 4331-4340.

[30] I. Leouifoudi, H. Harnafi, A. Zyad, Olive mill waste extracts: polyphenols content, antioxidant, and antimicrobial activities, Adv. Pharmacol. Sci. (2015) 11. Article ID 714138 .

[31] I. Dammak, M. Neves, H. Isoda, S. Sayadi, M. Nakajima, Recovery of polyphenols from olivemill wastewater using drowning-out crystallization based separation process, Innov. Food Sci. Emerg. Technol. 34 (2016) 326-335.

[32] E. Garcia-Castello, A. Cassano, A. Criscuoli, C. Conidi, E. Drioli, Recovery and concentration of polyphenols from olive mil wastewaters by integrated membrane system, Water Res. 44 (2010) 3883-3892. 